\title{
Injury of the Lower Ascending Reticular Activating System by Subfalcine Herniation in a Patient With a Cerebral Infarct
}

\author{
Sung Ho Jang, MD, Young Hyeon Kwon, BS \\ Department of Physical Medicine and Rehabilitation, Yeungnam University College of Medicine, Daegu, Korea
}

In the neurosurgery department of a university hospital a 47-year-old male patient underwent decompressive craniectomy in the left fronto-parieto-temporal area. This was due to brain swelling and subfalcine herniation following a cerebral infarction in the left middle cerebral artery region (Fig. 1A, 1B). Two weeks after its onset he was admitted to the rehabilitation department of another university hospital. The patient displayed impaired consciousness, with a Glasgow Coma Scale (GCS) score of 10 (eye opening, 4; best verbal response, 1; best motor response, 5) and a Coma Recovery Scale-Revised (CRSR) score of 12 (auditory function, 2; visual function, 4; motor function, 3; verbal function, 1; communication, 0 ; arousal, 2). The patient underwent comprehensive rehabilitative therapy including drug therapy for impaired consciousness (methylphenidate $20 \mathrm{mg}$, amantadine 200 $\mathrm{mg}$, levodopa $250 \mathrm{mg}$, pramipexole $0.375 \mathrm{mg}$, ropinirole $1.25 \mathrm{mg}$, venlafaxine $75 \mathrm{mg}$ ) [1]. After 2 weeks of rehabilitation, his GCS and CRS-R scores recovered to full scores of 15 and 23, respectively.

Diffusion tensor imaging data were acquired 2 weeks after onset by using a Philips Gyroscan Intera 1.5T (Philips Medical Systems, Cleveland, OH, USA) and fiber tracking was performed by utilizing a probabilistic tractogra- phy method. The ascending reticular activating system (ARAS) was divided into three components: (1) the lower dorsal ARAS-between the pontine reticular formation and the thalamic intralaminar nucleus; (2) the lower ventral ARAS-between the pontine reticular formation and the hypothalamus; (3) the upper ARAS-between the thalamic intralaminar nucleus and the cerebral cortex [2]. The right lower ventral ARAS showed severe narrowing, whereas the lower portions of the lower dorsal ARAS and the left lower ventral ARAS revealed mild narrowing. The upper ARAS displayed decreased neural connectivity in the left hemisphere, particularly in the basal forebrain and the prefrontal cortex (Fig. 1C).

In this study, at two weeks after cerebral infarction, diffusion tensor tractography (DTT) of the ARAS revealed severe injury of the right lower ventral ARAS and the left upper ARAS, and mild injury of the lower dorsal ARAS and the left ventral ARAS. The results suggested that the lower dorsal and ventral ARAS were injured as a result of subfalcine herniation following cerebral infarction [3]. The patient showed rapid recovery of consciousness, reaching a normal state after two weeks of rehabilitation that included drug therapy for impaired consciousness [1]. Notably, the patient's impaired consciousness 

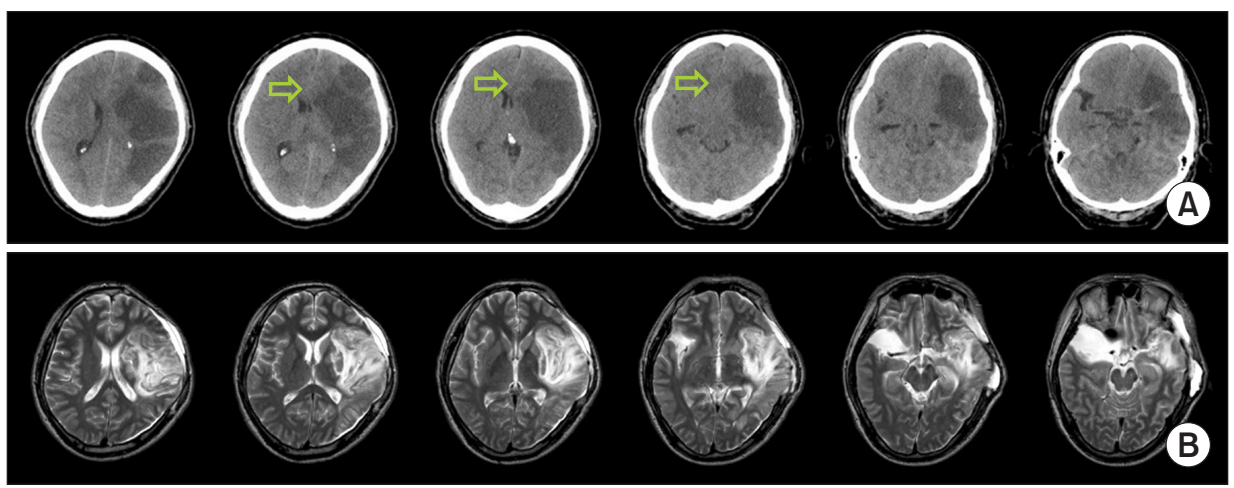

Patient
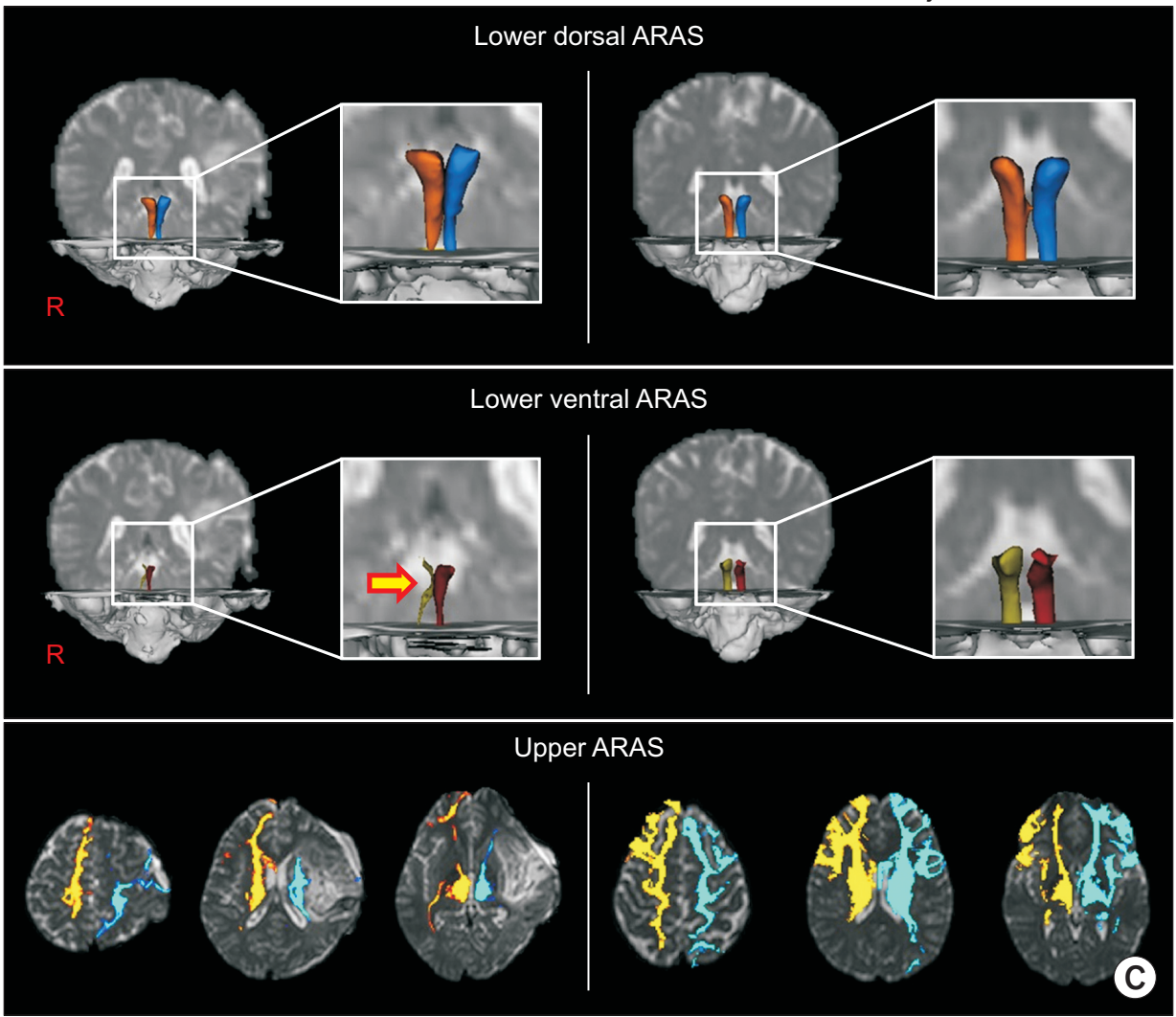

Fig. 1. Brain computed tomography images (A) at 3 days after onset show subfalcine herniation (arrows), and brain magnetic resonance images (B) at 2 weeks after onset show a leukomalactic lesion in the left middle cerebral artery area. (C) Results of diffusion tensor tractography for the ascending reticular activating system (ARAS) of the patient and a normal subject (48-year-old female). The right lower ventral ARAS shows severe narrowing (arrow) whereas the lower portions of the lower dorsal ARAS and the left lower ventral ARAS reveal mild narrowing. The upper ARAS showed decreased neural connectivity in the left hemisphere. showed a good response to medication especially methylphenidate. Based on these results, we suggest the patient might have impaired consciousness and hypersomnia because the main lesion in his ARAS was located in the right lower ventral ARAS, a region related to hypersomnia following brain injury [4]. This case study suggests that an accurate diagnosis based on the state of ARAS on DTT would be helpful in achieving successful rehabilitation in patients with impaired consciousness following acute brain injury.

\section{CONFLICT OF INTEREST}

No potential conflict of interest relevant to this article was reported.

\section{ACKNOWLEDGMENTS}

This work was supported by the National Research Foundation (NRF) of Korea Grant funded by the Korean Government (MSIP) (No. 2015R1A2A2A01004073). 


\section{REFERENCES}

1. Ciurleo R, Bramanti P, Calabro RS. Pharmacotherapy for disorders of consciousness: are 'awakening' drugs really a possibility? Drugs 2013;73:1849-62.

2. Jang SH, Lee HD. Ascending reticular activating system recovery in a patient with brain injury. Neurology 2015;84:1997-9.
3. Jang SH, Kim SH, Seo YS. Injury of the ascending reticular activating system by subfalcine herniation after subdural hematoma: a case report. Am J Phys Med Rehabil 2016;95:e129-30.

4. Jang SH, Chang CH, Jung YJ, Kwon HG. Hypersomnia due to injury of the ventral ascending reticular activating system following cerebellar herniation: a case report. Medicine (Baltimore) 2017;96:e5678. 